\title{
Approximate Controllability of Fractional Integrodifferential Evolution Equations
}

\author{
R. Ganesh, ${ }^{1}$ R. Sakthivel, ${ }^{2}$ N. I. Mahmudov, ${ }^{3}$ and S. M. Anthoni ${ }^{1}$ \\ ${ }^{1}$ Department of Mathematics, Anna University, Regional Centre, Coimbatore 641 047, Tamil Nadu, India \\ ${ }^{2}$ Department of Mathematics, Sungkyunkwan University, Suwon 440-746, Republic of Korea \\ ${ }^{3}$ Department of Mathematics, Eastern Mediterranean University, Gazimagusa, Mersin 10, Turkey
}

Correspondence should be addressed to R. Sakthivel; krsakthivel@yahoo.com

Received 11 March 2013; Revised 7 May 2013; Accepted 7 May 2013

Academic Editor: Naseer Shahzad

Copyright (C) 2013 R. Ganesh et al. This is an open access article distributed under the Creative Commons Attribution License, which permits unrestricted use, distribution, and reproduction in any medium, provided the original work is properly cited.

\begin{abstract}
This paper addresses the issue of approximate controllability for a class of control system which is represented by nonlinear fractional integrodifferential equations with nonlocal conditions. By using semigroup theory, $p$-mean continuity and fractional calculations, a set of sufficient conditions, are formulated and proved for the nonlinear fractional control systems. More precisely, the results are established under the assumption that the corresponding linear system is approximately controllable and functions satisfy nonLipschitz conditions. The results generalize and improve some known results.
\end{abstract}

\section{Introduction}

In recent years, use theory of fractional calculus and fractional differential equations has gained importance and popularity due to its applications in various fields of science and engineering. Various physical phenomena in science and engineering can be successfully modeled by using fractional calculus theory. Due to its tremendous scope and applications, several papers have been devoted to study the existence of mild solutions of fractional differential equations (see [1$4]$ and references therein). On the other hand, controllability is an important property of a control system which plays an important role in the analysis and design of control systems [5-8]. Most literatures in this direction so far have been concerned with controllability of nonlinear differential equations in infinite-dimensional spaces without fractional derivatives (see [9] and references therein). Using generalized open mapping theorem, a set of sufficient conditions for constrained local relative controllability near the origin are formulated and proved for the semilinear systems with delayed controls in $[10,11]$.

Recently, only few papers deal with the controllability of fractional dynamical systems $[12-14]$. Klamka $[15,16]$ derived a set of sufficient conditions for the local controllability of finite-dimensional fractional discrete-time semilinear systems. However, the problem of controllability for fractional systems has not been fully investigated, and there is still room open for further research in this area [17]. Moreover, the approximate controllable systems are more prevalent, and very often, approximate controllability is completely adequate in applications (see [18-21] and references therein). Therefore, it is important, in fact necessary, to study the weaker concept of controllability, namely, approximate controllability for nonlinear fractional integrodifferential systems. Motivated by this fact, in this paper, we consider the approximate controllability of the fractional nonlinear integrodifferential evolution equations with nonlocal initial condition in the following form:

$$
\begin{gathered}
{ }^{C} D^{q} x(t)=-A x(t)+B u(t)+t^{n} f(t, x(t),(H x)(t)), \\
t \in J=[0, b], \quad n \in Z^{+}, \quad q \in(0,1), \\
x(0)=g(x)+x_{0} \in X_{\gamma},
\end{gathered}
$$

where the state $x(\cdot)$ takes the values in a Hilbert space $X$, ${ }^{C} D^{q}$ denotes Caputo derivative, $-A: D(A) \rightarrow X$ is the infinitesimal generator of an analytic semigroup $\{T(t), t \geq 0\}$ on $X$; the control function $u(\cdot)$ is given in $L^{2}(J, U)$; $U$ is a 
Hilbert space; $B$ is a bounded linear operator from $U$ into $X$; the operator $H$ is defined by $(H x)(t)=\int_{0}^{b} h(t, s, x(s)) d s$; the nonlinear term $f: J \times X_{\gamma} \times X_{\gamma} \rightarrow X\left(\right.$ or $\left.X_{\gamma}\right)$ is a given function, where here $J=[0, b]$; and $X_{\gamma}=D\left(A^{\gamma}\right)(0<\gamma<1)$ is a Hilbert space with the norm $\|x\|_{\gamma}=\left\|A^{\gamma} x\right\|$ for $x \in X_{\gamma}$. The functions $f, h$, and $g$ will be specified later. In fact, our results in this paper are motivated by the recent work of [20], and the fractional integrodifferential equations are studied in [4]. The main objective of this paper is to derive conditions for the approximate controllability of (1) with non-Lipschitz coefficients, and the associated linear system is approximately controllable.

\section{Preliminaries}

In this section, we provide definitions, lemmas, and notations necessary to establish our main results [4]. Let $\|f\|_{L^{p}\left(J, R^{+}\right)}$ denote the $L^{p}\left(J, R^{+}\right)$norm of $f$ whenever $f \in L^{p}\left(J, R^{+}\right)$ for some $p$ with $1<p<\infty$. Let $\mathscr{C}_{\gamma}$ denote the Banach space $C\left(J, X_{\gamma}\right)$ endowed with sup norm given by $\|x\|_{\infty} \equiv$ $\sup _{t \in J}\|x\|_{\gamma}$, for $x \in \mathscr{C}_{\gamma}, \gamma \in(0,1)$.

Let us recall the following known results.

The fractional integral of order $\beta$ with the lower limit 0 for a function $f$ is defined as

$$
I^{\beta} f(t)=\frac{1}{\Gamma(\beta)} \int_{0}^{t} \frac{f(s)}{(t-s)^{1-\beta}} d s, \quad t>0, \beta>0,
$$

provided the right-hand side is pointwise defined on $[0, \infty)$, where $\Gamma(\cdot)$ is the gamma function.

Riemann-Liouville derivative of order $\beta$ with lower limit zero for a function $f:[0, \infty) \rightarrow R$ can be written as

$$
\begin{array}{r}
{ }^{L} D^{\beta} f(t)=\frac{1}{\Gamma(n-\beta)} \frac{d^{n}}{d t^{n}} \int_{0}^{t} \frac{f(s)}{(t-s)^{\beta+1-n}} d s, \\
t>0, \quad n-1<\beta<n .
\end{array}
$$

The Caputo derivative of order $\beta$ for a function $f:[0$, $\infty) \rightarrow R$ can be written as

$$
\begin{array}{r}
D^{\beta} f(t)={ }^{L} D^{\beta}\left(f(t)-\sum_{k=0}^{n-1} \frac{t^{k}}{k !} f^{(k)}(0)\right), \\
t>0, \quad n-1<\beta<n .
\end{array}
$$

Remark 1 (see [4]). (i) If $f(t) \in C^{n}[0, \infty)$, then

$$
\begin{array}{r}
D^{\beta} f(t)=\frac{1}{\Gamma(n-\beta)} \int_{0}^{t} \frac{f^{n}(s)}{(t-s)^{\beta+1-n}} d s=I^{n-\beta} f^{n}(s), \\
t>0, \quad 0 \leq n-1<\beta<n .
\end{array}
$$

(ii) The Caputo derivative of a constant is equal to zero.

(iii) If $f$ is an abstract function with values in $X$, then integrals which appear in the above results are taken in Bochner's sense.

For additional details concerning the fractional derivative, we refer the reader to [3].
To define the mild solution for the control system (1), we associate problem (1) to the following integral equation [4]:

$$
\begin{array}{r}
x(t)=\mathscr{T}(t)\left[x_{0}+g(x)\right]+\int_{0}^{t}(t-s)^{q-1} \mathcal{S}(t-s) B u(s) d s \\
+\int_{0}^{t}(t-s)^{q-1} s^{n} \mathcal{S}(t-s) f(s, x(s),(H x)(s)) d s, \\
t \in J,
\end{array}
$$

where $\mathscr{T}(t)=\int_{0}^{\infty} \xi_{q}(\theta) T\left(t^{q} \theta\right) d \theta, \mathcal{S}(t)=q \int_{0}^{\infty} \theta \xi_{q}(\theta) T\left(t^{q} \theta\right) d \theta$, $\xi_{q}(\theta)=(1 / q) \theta^{-1-1 / q} \bar{w}_{q}\left(\theta^{-1 / q}\right) \geq 0, \bar{w}_{q}(\theta)=(1 /$ $\pi) \sum_{n=1}^{\infty}(-1)^{n-1} \theta^{-q n-1}(\Gamma(n q+1) / n !) \sin (n \pi q), \theta \in(0, \infty)$, and $\xi_{q}$ is a probability density function defined on $(0, \infty)$; that is $\xi_{q}(\theta) \geq 0, \theta \in(0, \infty)$, and $\int_{0}^{\infty} \xi_{q}(\theta) d \theta=1$.

Definition 2. A function $x\left(\cdot ; x_{0}, u\right) \in C\left(J, X_{\gamma}\right)$ is said to be a mild solution of (1) if for any $u(\cdot) \in L^{2}(J, U)$ the integral equation (6) is satisfied.

Definition 3. The system (1) is said to be approximately controllable on the interval $J$ if $\overline{\Re\left(b, x_{0}\right)}=X$, where

$$
\mathfrak{R}\left(b, x_{0}\right)=\left\{x_{b}\left(x_{0} ; u\right)(0): u(\cdot) \in L^{2}(J, U)\right\}
$$

is called the reachable set of system (1) at terminal time $b$, and its closure in $X$ is denoted by $\overline{\Re\left(b, x_{0}\right)}$; let $x_{b}\left(x_{0} ; u\right)$ be the state value of (1) at terminal time $b$ corresponding to the control $u$ and the initial value $x_{0} \in X$.

Consider the following linear fractional differential control system

$$
\begin{gathered}
D_{t}^{q} x(t)=A x(t)+B u(t), \quad t \in J=[0, b], 0<q<1, \\
x(0)=x_{0} .
\end{gathered}
$$

The approximate controllability for the system (8) is a natural generalization of approximate controllability for the linear first order control system (see [18]). It is convenient at this point to introduce the controllability operator associated with the linear system

$$
\Gamma_{0}^{b}=\int_{0}^{b}(b-s)^{q-1} \mathcal{S}(b-\tau) B B^{*} \mathcal{S}^{*}(b-\tau) d \tau,
$$

where $B^{*}$ denotes the adjoint of $B$ and $\mathcal{S}^{*}(t)$ is the adjoint of $\delta(t)$. It is straightforward that the operator $\Gamma_{0}^{b}$ is a linear bounded operator. Let $R\left(\alpha, \Gamma_{0}^{b}\right)=\left(\alpha I+\Gamma_{0}^{b}\right)^{-1}$ for $\alpha>0$.

Lemma 4. The linear fractional control system (8) is approximately controllable on $J$ if and only if $\alpha R\left(\alpha, \Gamma_{0}^{b}\right) \rightarrow 0$ as $\alpha \rightarrow 0^{+}$in the strong operator topology.

The proof of this lemma is a straightforward adaptation of the proof of Theorem 2 of [18]. 
Lemma 5 (see [4]). For each $\psi \in L^{p}(J, X)$ with $1 \leq p<$ $+\infty$, one has $\lim _{h \rightarrow 0} \int_{0}^{b}\|\psi(t+h)-\psi(t+h)\|^{p} d t=0$, where $\psi(s)=0$ for $s$ does not belong to $J$.

Lemma 6 (see [4]). The operators $\mathscr{T}$ and $\mathcal{S}$ have the following properties.

(i) For fixed $t \geq 0, \mathscr{T}(t)$ and $\mathcal{S}(t)$ are linear and bounded operators. For any $x \in X$,

$$
\|\mathscr{T}(t) x\| \leq M\|x\|, \quad\|\mathcal{S}(t) x\| \leq \frac{M q}{\Gamma(q+1)}\|x\| .
$$

(ii) $\{\mathscr{T}(t), t \geq 0\}$ and $\{\mathcal{S}(t), \geq 0\}$ are strongly continuous.

(iii) For every $t>0, \mathscr{T}(t)$ and $\mathcal{S}(t)$ are also compact operators.

(iv) For any $x \in X, \beta \in(0,1)$, and $\gamma \in(0,1)$ one has

$$
\begin{gathered}
A \mathcal{S}(t) x=A^{1-\beta} \mathcal{S}(t) A^{\beta} x, \quad t \in J, \\
\left\|A^{\gamma} \mathcal{S}(t)\right\| \leq \frac{M_{\gamma} q \Gamma(2-\gamma)}{\Gamma(1+q(1-\gamma))} t^{-\gamma q}, \quad 0<t \leq b .
\end{gathered}
$$

(v) For fixed $t \geq 0$ and any $x \in X_{\gamma}$, one has

$$
\|\mathscr{T}(t) x\|_{\gamma} \leq M\|x\|_{\gamma}, \quad\|\delta(t) x\|_{\gamma} \leq \frac{M q}{\Gamma(q+1)}\|x\|_{\gamma} .
$$

(vi) $\mathscr{T}_{\gamma}(t)$ and $\mathcal{S}_{\gamma}(t), t>0$ are uniformly continuous; that is, for each fixed $t>0$ and $\epsilon>0$, there exists $h>0$ such that

$\left\|\mathscr{T}_{\gamma}(t+\epsilon)-\mathscr{T}_{\gamma}(t)\right\|_{\gamma}<\epsilon, \quad$ for $t+\epsilon \geq 0, \quad|\epsilon|<h$,

$\left\|\mathcal{S}_{\gamma}(t+\epsilon)-\mathcal{S}_{\gamma}(t)\right\|_{\gamma}<\epsilon, \quad$ for $t+\epsilon \geq 0,|\epsilon|<h$,

where $\mathscr{T}_{\gamma}(t)=\int_{0}^{\infty} \xi_{q}(\theta) T_{\gamma}\left(t^{q} \theta\right) d \theta, \quad \delta_{\gamma}(t)=$ $q \int_{0}^{\infty} \theta \xi_{q}(\theta) T_{\gamma}\left(t^{q} \theta\right) d \theta$.

\section{Main Result}

In this section, we present our main result on approximate controllability of control system (1). We prove that under certain conditions, approximate controllability of the linear system (8) implies the approximate controllability of nonlinear fractional system (1). In order to establish the result, we require the following assumptions.

$\left(H_{1}\right)$ The function $f: J \times X_{\gamma} \times X_{\gamma} \rightarrow X_{\gamma}$ is Carathéodory, and there exists a positive function $\rho \in L^{p}\left(J, R^{+}\right)$for some $p$ with $1<p<\infty$ such that $\|f(t, x, y)\|_{\gamma} \leq \rho(t)$ for all $x, y \in X_{\gamma}$ and $t \in J$.

$\left(H_{2}\right)$ The function $g: \mathscr{C}_{\gamma} \rightarrow X_{\gamma}$ is completely continuous, and there exist $\beta_{1}, \beta_{2}>0$ such that

$$
\|g(x)\|_{\gamma} \leq \beta_{1}\|x\|_{\infty}+\beta_{2} .
$$

$\left(H_{3}\right)$ The function $h: D_{h} \times X_{\gamma} \rightarrow X_{\gamma}$ is continuous, and there exist $L_{1}, L_{2}>0$ such that $\| h(t, s, x)-$ $h(t, s, y)\left\|_{\gamma} \leq L_{1}\right\| x-y \|_{\gamma}+L_{2}$ for each $(t, s) \in D_{h}=$ $\left\{(t, s) \in R^{2}, 0 \leq s, t \leq b\right\}$ and $x, y \in X_{\gamma}$.

$\left(H_{4}\right)$ The linear fractional control system (8) is approximately controllable.

$\left(H_{5}\right)$ The function $f: J \times X_{\gamma} \times X_{\gamma} \rightarrow X_{\gamma}$ is continuous and uniformly bounded, and there exists $N>0$ such that $\|f(t, x, y)\| \leq N$ for all $(t, x, y) \in J \times X_{\gamma} \times X_{\gamma}$.

$\left(H_{6}\right)$ The semigroup $T(t)$ is compact.

In order to prove the required result, for $\alpha>0$, we define the operator $F_{\alpha}$ on $C\left(J, X_{\gamma}\right)$ as

$$
\left(F_{\alpha} x\right)(t)=z(t),
$$

where

$$
\begin{gathered}
z(t)=\mathscr{T}(t)\left[x_{0}+g(x)\right]+\int_{0}^{t}(t-s)^{q-1} \mathcal{S}(t-s) B v(s) d s \\
+\int_{0}^{t}(t-s)^{q-1} s^{n} \mathcal{S}(t-s) f(s, x(s),(H x)(s)) d s \\
t \in J \\
v(t)=B^{*} \mathcal{S}^{*}(b-t) R\left(\alpha, \Gamma_{0}^{b}\right) p(x(\cdot)), \\
p(x(\cdot))=x_{b}-\mathscr{T}(b)\left[x_{0}+g(x)\right] \\
-\int_{0}^{b}(b-s)^{q-1} s^{n} \mathcal{S}(b-s) \\
\times f(s, x(s),(H x)(s)) d s .
\end{gathered}
$$

Theorem 7. Under the conditions $\left(H_{1}\right),\left(H_{2}\right),\left(H_{3}\right)$, and $\left(H_{6}\right)$, the control system (1) admits a mild solution on $[0, b]$, and here $M_{B}=\|B\|$ and $M_{1}=\|\rho\|_{L^{p^{2}}\left(J, R^{+}\right)}$.

Proof. The main aim in this section is to find conditions for solvability of systems (16) and (17) for $\alpha>0$. In the Banach space $C\left(J, X_{\gamma}\right)$, consider a set

$$
B_{r_{0}}=\left\{x(\cdot) \in C\left(J, X_{\gamma}\right):\|x\|_{\gamma} \leq r\right\},
$$

where $r$ is the positive constant. Now, it will be shown that, using Schauder's fixed point theorem, for all $\alpha>0$, the operator $F_{\alpha}: C\left(J, X_{\gamma}\right) \rightarrow C\left(J, X_{\gamma}\right)$ has a fixed point.

First, we prove that for an arbitrary $\alpha>0$ and there is a positive constant $r_{0}=r_{0}(\alpha)$ such that $F_{\alpha}: B_{r_{0}} \rightarrow B_{r_{0}}$. 
Let $x \in B_{r_{0}}$, and then, for $t \in J$ and $1 / p<q(\Rightarrow(p q-1))>$ 0 , using Holders inequality, Lemma 6 , and conditions $\left(H_{1}\right)$ and $\left(H_{2}\right)$ in (17), we obtain

$$
\begin{aligned}
&\|v(t)\|_{\gamma} \leq\left\|B^{*} \mathcal{S}^{*}(b-t) R\left(\alpha, \Gamma_{0}^{b}\right)\right\| \\
& \times\left[\left\|x_{b}\right\|_{\gamma}-\left\|\mathcal{T}(b)\left[x_{0}+g(x)\right]\right\|_{\gamma}\right. \\
& \quad+\int_{0}^{b}(b-s)^{q-1} s^{n} \\
&\left.\times\|\mathcal{S}(b-s) f(s, x(s),(H x)(s))\|_{\gamma} d s\right] \\
& \leq \frac{M_{B} M q}{\alpha \Gamma(q+1)}\left[\left\|x_{b}\right\|_{\gamma}+M\left(\left\|x_{0}\right\|_{\gamma}+\beta_{1}\|x\|_{\infty}+\beta_{2}\right)\right. \\
& \quad+\frac{M_{1} M q}{\Gamma(q+1)}\left(\frac{p-1}{p q-1}\right)^{(p-1) / p} \\
&\left.\times\left(\frac{p-1}{n p^{2}+p-1}\right)^{(p-1) / p^{2}} b^{n+q-1 / p^{2}}\right] .
\end{aligned}
$$

It follows from (16) that

$$
\begin{aligned}
\|z(t)\|_{\gamma} \leq & \left\|\mathscr{T}(t)\left[x_{0}+g(x)\right]\right\|_{\gamma} \\
& +\int_{0}^{t}(t-s)^{q-1}\|\mathcal{S}(t-s) B v(s)\|_{\gamma} d s \\
& +\int_{0}^{t}(t-s)^{q-1} s^{n}\|\mathcal{S}(t-s) f(s, x(s),(H x)(s))\|_{\gamma} d s \\
\leq & M\left(\left\|x_{0}\right\|_{\gamma}+\beta_{1}\|x\|_{\infty}+\beta_{2}\right) \\
& +\frac{M_{B} M q}{\Gamma(q+1)}\left(\frac{p-1}{q p-1}\right)^{(p-1) / p} b^{((q-1) p+p-1) / p} b\|v(s)\|_{\gamma} \\
& +\frac{M_{1} M q}{\Gamma(q+1)}\left(\frac{p-1}{p q-1}\right)^{(p-1) / p}\left(\frac{p-1}{n p^{2}+p-1}\right)^{(p-1) / p^{2}} \\
& \times b^{n+q-1 / p^{2}} .
\end{aligned}
$$

From the above two inequalities, we get that $\left\|\left(F_{\alpha} x\right)(t)\right\|_{\gamma} \leq r_{0}$. This follows that $F_{\alpha}$ maps $B_{r_{0}}$ into itself.

For each $\alpha>0$, we prove the operator $F_{\alpha}$ maps $B_{r_{0}}$ into a relatively compact subset of $B_{r_{0}}$. First, we show that $V(t)=$ $\left\{\left(F_{\alpha} x\right)(t): x \in B_{r_{0}}\right\}$ is relatively compact in $X_{\gamma}$ for every $t \in J$. The case $t=0$ is obvious. Let $t \in(0, b]$ be fixed, and for each $\varepsilon \in(0, t)$, arbitrary $\delta>0$, and $x \in B_{r_{0}}$, we define the operator $F_{\alpha}^{\varepsilon, \delta}$ by

$$
\begin{aligned}
& \left(F_{\alpha}^{\varepsilon, \delta} x\right)(t) \\
& =\mathscr{T}\left[x_{0}+g(x)\right] \\
& +q \int_{0}^{t-\varepsilon} \int_{\delta}^{\infty} \theta(t-s)^{q-1} \xi_{q}(\theta) T\left((t-s)^{q} \theta\right) \\
& \quad \times B v(s) d \theta d s \\
& +q \int_{0}^{t-\varepsilon} \int_{\delta}^{\infty} \theta(t-s)^{q-1} s^{n} \xi_{q}(\theta) T\left((t-s)^{q} \theta\right) \\
& =T\left(\varepsilon^{q} \delta\right) \quad \times f(s, x(s),(H x)(s)) d \theta d s \\
& \quad \times \int_{\delta}^{\infty} \xi_{q}(\theta)\left(T\left(t^{q} \theta\right)-T\left(\varepsilon^{q} \delta\right)\right) d \theta\left[x_{0}+g(x)\right] \\
& +\left(T\left(\varepsilon^{q} \delta\right)\right) q \\
& \quad \times \int_{0}^{t-\varepsilon} \int_{\delta}^{\infty} \theta(t-s)^{q-1} \xi_{q}(\theta) \\
& \quad \times\left(T\left((t-s)^{q} \theta\right)-T\left(\varepsilon^{q} \delta\right)\right) B v(s) d \theta d s \\
& +T\left(\varepsilon^{q} \delta\right) q \int_{0}^{t-\varepsilon} \int_{\delta}^{\infty} \theta(t-s)^{q-1} s^{n} \xi_{q}(\theta) \\
& \times\left(T\left((t-s)^{q} \theta\right)-T\left(\varepsilon^{q} \delta\right)\right) \\
& \times\left(\varepsilon^{q} \delta\right) y(t, \varepsilon) . \\
& \times f(s, x(s),(H x)(s)) d \theta d s
\end{aligned}
$$

Since $T\left(\varepsilon^{q} \delta\right)$ is compact in $X_{\gamma}$ and $y(t, \varepsilon)$ is bounded in $B_{r_{0}}$, the set $V_{\varepsilon}(t)=\left\{\left(F_{\alpha}^{\varepsilon, \delta} x\right)(t): x \in B_{r_{0}}\right\}$ is relatively compact in $X_{\gamma}$ [4]. On the other hand, using $\left(H_{1}\right)$ and Holder's inequality, we have

$$
\begin{aligned}
& \left\|\left(F_{\alpha} x\right)(t)-\left(F_{\alpha}^{\varepsilon, \delta} x\right)(t)\right\|_{\gamma} \\
& \leq q \int_{0}^{t} \int_{0}^{\delta} \theta(t-s)^{q-1} \xi_{q}(\theta) \\
& \quad \times\left\|T\left((t-s)^{q} \theta\right)\left[B v(s)+s^{n} f(s, x(s),(H x)(s))\right]\right\|_{\gamma} d \theta d s \\
& +q \int_{t-\varepsilon}^{t} \int_{\delta}^{\infty} \theta(t-s)^{q-1} \xi_{q}(\theta) \\
& \quad \times\left\|T\left((t-s)^{q} \theta\right)\left[B v(s)+s^{n} f(s, x(s),(H x)(s))\right]\right\|_{\gamma} d \theta d s \\
& \leq M_{B} M q\left(\frac{p-1}{q p-1}\right)^{(p-1) / p} t^{1+q-1 / p}\|v(s)\|_{\gamma} \int_{0}^{\delta} \theta \xi_{q}(\theta) d \theta
\end{aligned}
$$




$$
\begin{aligned}
& +M_{1} M q\left(\frac{p-1}{p q-1}\right)^{(p-1) / p}\left(\frac{p-1}{n p^{2}+p-1}\right)^{(p-1) / p^{2}} b^{n+q-1 / p^{2}} \\
& \times \int_{0}^{\delta} \theta \xi_{q}(\theta) d \theta \\
& +\frac{M_{B} M q}{\Gamma(q+1)}\left(\frac{p-1}{q p-1}\right)^{(p-1) / p} \varepsilon^{1+q-1 / p}\|v(s)\|_{\gamma} \\
& +\frac{M_{1} M q}{\Gamma(q+1)}\left(\frac{p-1}{p q-1}\right)^{(p-1) / p}\left(\frac{p-1}{n p^{2}+p-1}\right)^{(p-1) / p^{2}} \varepsilon^{n+q-1 / p^{2}} .
\end{aligned}
$$

This implies that there are relatively compact sets $V_{\varepsilon}(t)$ arbitrarily close to the set $V(t)$ for each $t \in(0, b]$. Thus, $V(t)$ is relatively compact in $X_{\gamma}$ for all $t \in(0, b]$ since it is compact at $t=0$. Therefore, we have the relatively compactness in $X_{\gamma}$ for all $t \in[0, b]$.

Next we show that $V(t)=\left\{\left(F_{\alpha} x\right)(t): x \in B_{r_{0}}\right\}$ is an equicontinuous family of functions on $[0, b]$. By the compactness of the set, we can prove that the functions $F_{\alpha} x, x \in B_{r_{0}}$ are equicontinuous at $t=0$. For any $x \in B_{r_{0}}$ and $0<t_{1}<t_{2} \leq$ $b$, we have

$$
\begin{aligned}
& \left\|z\left(t_{2}\right)-z\left(t_{1}\right)\right\|_{\gamma} \\
& \leq\left\|\left(\mathscr{T}\left(t_{2}\right)-\mathscr{T}\left(t_{1}\right)\right)\left[x_{0}+g(x)\right]\right\|_{\gamma} \\
& +\left\|\int_{0}^{t_{1}}\left(t_{2} s\right)^{q-1}\left[\mathcal{S}\left(t_{2}-s\right)-\mathcal{S}\left(t_{1}-s\right)\right] B v(s) d s\right\|_{\gamma} \\
& +\left\|\int_{0}^{t_{1}}\left[\left(t_{2}-s\right)^{q-1}-\left(t_{1}-s\right)^{q-1}\right] \mathcal{S}\left(t_{1}-s\right) B v(s) d s\right\|_{\gamma} \\
& +\left\|\int_{t_{1}}^{t_{2}}\left(t_{2}-s\right)^{q-1} \mathcal{S}\left(t_{2}-s\right) B v(s) d s\right\|_{\gamma} \\
& \quad+\| \int_{0}^{t_{1}}\left(t_{2}-s\right)^{q-1} s^{n}\left[\mathcal{S}\left(t_{2}-s\right)-\mathcal{S}\left(t_{1}-s\right)\right] \\
& \quad \times f(s, x(s),(H x)(s)) d s \|_{\gamma} \\
& \quad+\| \int_{0}^{t_{1}}\left[\left(t_{2}-s\right)^{q-1}-\left(t_{1}-s\right)^{q-1}\right] s^{n} \mathcal{S}\left(t_{2}-s\right) \\
& \quad+\left\|\int_{t_{1}}^{t_{2}}\left(t_{2}-s\right)^{q-1} s^{n} \mathcal{S}\left(t_{2}-s\right) f(s, x(s),(H x)(s)) d s\right\|_{\gamma} \\
& \leq I_{1}+I_{2}+I_{3}+I_{4}+I_{5}+I_{6}+I_{7} .
\end{aligned}
$$

Now, we have to prove that $I_{1}, I_{2}, I_{3}, I_{4}, I_{5}, I_{6}$, and $I_{7}$ tend to 0 independently of $x \in B_{r_{0}}$ as $t_{2}-t_{1} \rightarrow 0$. By Lemma 6 , one can easily show that $\lim _{t_{1} \rightarrow t_{2}} I_{1}=0$. By using Lemmas 5 and
6 and the Lagrange mean value theorem and following the similar procedure as in the proof of Theorem 3.2 of [4], one can deduce that $\lim _{t_{1} \rightarrow t_{2}} I_{5}, I_{6}, I_{7}=0$. In a similar way, we can obtain

$$
\begin{aligned}
I_{2} \leq & \frac{\Gamma(2)}{\Gamma(q+1)} \frac{M_{1+\gamma}}{\gamma}\left(\frac{(p-1)^{2}}{(q-1) p^{2}+(p-1)^{2}}\right)^{(p-1)^{2} / p^{2}} \\
& \times b^{\left((q-1) p^{2}+(p-1)^{2}\right) / p^{2}} \\
& \times\left(\int_{0}^{b}\left[\left(t_{2}-s\right)^{-q \gamma}-\left(t_{1}-s\right)^{-q \gamma}\right]^{p^{2} /(p-1)} d s\right)^{(p-1) / p^{2}} \\
& \times b M_{B}\|v(s)\|, \\
I_{3} \leq & \frac{M q}{\Gamma(q+1)}\left(\int_{0}^{b}\left[\left(t_{2}-s\right)^{q-1}-\left(t_{1}-s\right)^{q-1}\right]^{p /(p-1)} d s\right)^{(p-1) / p} \\
& \times b M_{B}\|v(s)\|, \\
I_{4} \leq & \frac{M q}{\Gamma(q+1)}\left(\frac{p-1}{p q-1}\right)^{(p-1) / p}\left(t_{2}-t_{1}\right)^{((q-1) p+p-1) / p} \\
& \times M_{B}\|v(s)\|\left(t_{2}-t_{1}\right) .
\end{aligned}
$$

By the Lagrange mean value theorem and Lemmas 5 and 6 , it can be easily seen that $I_{2}, I_{3}$, and $I_{4}$ tend to 0 as $t_{2}-t_{1} \rightarrow 0$. Thus, the right-hand side of (23) tends to 0 as $t_{2}-t_{1} \rightarrow 0$ which means that $\left\{\left(F_{\alpha} x\right): x \in B_{r_{0}}\right\}$ is a family of equicontinuous functions. It can be easily seen that for all $\alpha>0, F_{\alpha}$ is continuous on $C\left(J, X_{\gamma}\right)$. Hence, by Arzela-Ascoli's theorem, $F_{\alpha}$ is compact. By Schauder's fixed point theorem, $F_{\alpha}$ has a fixed point $x \in B_{r_{0}}$. Thus, the control system (1) has at least one mild solution on $[0, b]$.

Theorem 8. Assume that $\left(H_{1}\right)-\left(H_{6}\right)$ hold. Then, system (1) is approximately controllable on $[0, b]$.

Proof. Linear system (8) is approximately controllable, by Lemma 4 , and it can be seen that approximate controllability of (8) is equivalent to convergence of the operator $\alpha R\left(\alpha, \Gamma_{0}^{b}\right)$ to zero operator in the strong operator topology as $\alpha \rightarrow 0^{+}$. Let $\widehat{x}_{\alpha}(\cdot)$ be a fixed point of $F_{\alpha}$ in $B_{r}$. Any fixed point of $F_{\alpha}$ is a mild solution of (1) under the control $\widehat{u}_{\alpha}(t)=B^{*} \mathcal{S}^{*}(b-$ t) $R\left(\alpha, \Gamma_{0}^{b}\right) p\left(\widehat{x}_{\alpha}\right)$ and satisfies $\widehat{x}_{\alpha}(b)=x_{b}-\alpha R\left(\alpha, \Gamma_{0}^{b}\right) p\left(\widehat{x}_{\alpha}\right)$. By the condition $\left(H_{5}\right)$, we have $\int_{0}^{b}\left\|f\left(s, \widehat{x}_{\alpha}(s),\left(H \hat{x}_{\alpha}\right)(s)\right)\right\|^{2} d s \leq$ $b N^{2}$, and consequently, the sequence $\left\{f\left(s, \widehat{x}_{\alpha}(s),\left(H \hat{x}_{\alpha}\right)(s)\right)\right\}$ is bounded in $L^{2}\left(J, X_{\gamma}\right)$. Then there is a subsequence denoted by $\left\{f\left(s, \widehat{x}_{\alpha}(s),\left(H \hat{x}_{\alpha}\right)(s)\right)\right\}$ that converges weakly to say $f(s)$ in $L^{2}\left(J, X_{\gamma}\right)$. Now, the compactness of $\mathcal{S}(t)$ implies that 
$\mathcal{S}(b-s) f\left(s, \widehat{x}_{\alpha}(s),\left(H \widehat{x}_{\alpha}\right)(s)\right) \rightarrow \mathcal{S}(b-s) f(s)$ in $L^{2}\left(J, X_{\gamma}\right)$, and we obtain

$$
\begin{aligned}
& \left\|p\left(\hat{x}_{\alpha}\right)-w\right\| \\
& =\| \int_{0}^{b}(b-s)^{q-1} s^{n} \mathcal{S}(b-s) \\
& \quad \times\left[f\left(s, \widehat{x}_{\alpha}(s),\left(H \hat{x}_{\alpha}\right)(s)\right)-f(s)\right] d s \| \\
& \leq \sup _{0 \leq t \leq b} \| \int_{0}^{t}(t-s)^{q-1} s^{n} \mathcal{S}(t-s) \\
& \quad \times\left[f\left(s, \widehat{x}_{\alpha}(s),\left(H \hat{x}_{\alpha}\right)(s)\right)-f(s)\right] d s \| \\
& \longrightarrow 0 \quad \text { as } \alpha \longrightarrow 0^{+},
\end{aligned}
$$

where

$$
\begin{aligned}
w= & x_{b}-\mathscr{T}(b)\left[x_{0}+g\left(\hat{x}_{\alpha}\right)\right] \\
& -\int_{0}^{b}(b-s)^{q-1} \mathcal{S}(b-s) s^{n} f(s) d s .
\end{aligned}
$$

Then, we obtain

$$
\begin{aligned}
\left\|\widehat{x}_{\alpha}(b)-x_{b}\right\| \leq & \left\|\alpha R\left(\alpha, \Gamma_{0}^{b}\right)(w)\right\| \\
& +\left\|\alpha R\left(\alpha, \Gamma_{0}^{b}\right)\right\|\left\|p\left(\hat{x}_{\alpha}\right)-w\right\| \\
\leq & \left\|\alpha R\left(\alpha, \Gamma_{0}^{b}\right)(w)\right\|+\left\|p\left(\hat{x}_{\alpha}\right)-w\right\| .
\end{aligned}
$$

It follows from Lemma 4 and estimation (25) that $\| \widehat{x}_{\alpha}(b)-$ $x_{b} \| \rightarrow 0$ as $\alpha \rightarrow 0^{+}$. This proves the approximate controllability of (1). The proof is completed.

Example 9. As an application of the obtained theory, we consider a control system which is represented by fractional partial differential equation. Let $X=U=L^{2}([0,1])$, and let $A: D(A) \subset X \rightarrow X$ be the operator defined by $A z=-z^{\prime \prime}$ with domain

$D(A)=\left\{z \in L^{2}([0,1]): z, z^{\prime}\right.$ are absolutely continuous,

$$
\left.z^{\prime \prime} \in L^{2}([0,1]), z(0)=z(1)=0\right\} .
$$

Let $D\left(A^{1 / 2}\right)=\left\{z(\cdot) \in X: \sum_{n=1}^{\infty}\left(z, e_{n}\right) e_{n} \in X\right\}$, and let $X_{1 / 2}=D\left(A^{1 / 2},\|\cdot\|_{1 / 2}\right)$, where $\|\cdot\|_{1 / 2}=\left\|A^{1 / 2}\right\|_{2}$. For each $z \in D\left(A^{1 / 2}\right)$ and $\left\|A^{-1 / 2}\right\|=1$, the operator $A^{1 / 2}$ is given by $A^{1 / 2} z=\sum_{n=1}^{\infty} n^{2}\left(z, e_{n}\right) e_{n}$, where $e_{n}(y)=\sqrt{2} \sin (n y)$ and $0 \leq y \leq 1, n=1,2, \ldots$ is the orthogonal set of eigenvectors of $A$. It is well known that $-A$ generates a compact analytic semigroup of uniformly bounded linear operator.
Consider the fractional partial differential equation with control in the following form:

$$
\begin{aligned}
& \frac{\partial^{q}}{\partial t^{q}} x(t, y) \\
& =\frac{\partial^{2}}{\partial y^{2}} x(t, y)+\mu(t, y) \\
& +\frac{e^{-t}}{e^{t}+e^{-t}} \cos \left[x(t, y)+\int_{0}^{b} \cos (t s) x(s, y) d s\right]+e^{-t} \\
& x(t, 0)=x(t, 1)=0, \quad t>0,1], \quad y \in[0,1] \\
& x(0, y)=\sum_{i=0}^{\sigma} \int_{0}^{1} k_{1}(y, \tau) x\left(t_{i}, \tau\right) d \tau \\
& +\sum_{i=0}^{\sigma} \int_{0}^{1} k_{2}(y, \tau) \frac{\partial}{\partial \tau} x\left(t_{i}, \tau\right) d \tau
\end{aligned}
$$

where $0 \leq q \leq 1 ; \mu:[0,1] \times[0,1] \rightarrow[0,1]$ is continuous; $k_{1}, k_{2} \in L^{2}([0,1] \times[0,1]) ; \sigma \in N$.

Let $x(t)(y)=x(t, y)$, and define the bounded linear operator $B: U \rightarrow X$ by $(B u)(t)(y)=\mu(t, y), 0 \leq y \leq 1$. Let $C\left([0,1], X_{1 / 2}\right)$ be a Banach space equipped with sup norm. Further, we define the operators $f:[0,1] \times X_{1 / 2} \rightarrow X_{1 / 2}$ and $g: C\left([0,1], X_{1 / 2}\right) \rightarrow X_{1 / 2}$ by

$$
\begin{aligned}
& f(t, x(t),(H x)(t))(y) \\
& =\frac{e^{-t}}{e^{t}+e^{-t}} \cos \left[x(t)+\int_{0}^{b} \cos (t s) x(s) d s\right](y)+e^{-t}, \\
& g(x)(y)=\left(\sum_{i=0}^{\sigma}(\mathscr{K} x)\left(t_{i}\right)\right)(y), \quad \text { for } x \in C\left(J, X_{1 / 2}\right),
\end{aligned}
$$

where $(H x)(t)(y)=\int_{0}^{b} \cos (t s) x(s, y) d s,(\mathscr{K} \phi)(\tau)=\int_{0}^{1} k_{1}(y$, $\tau) \phi(\tau) d \tau+\int_{0}^{1} k_{2}(y, \tau)(\partial / \partial \tau) \phi(\tau) d \tau$ for all $\phi \in X_{1 / 2}$. Moreover, the linear fractional control system corresponding to (29) is approximately controllable. Thus, with the above choices of $A, B, f$, and $g$, the system (29) can be written to the abstract form of (1). Therefore, all the conditions of Theorem 8 are satisfied. Hence, by Theorem 8 , the fractional nonlinear integrodifferential system (29) is approximately controllable on $[0,1]$.

Note. The considered system (1) is of the more general form, and in particular, if functions $f$ and $h$ have various physical meanings, it is important to note that (1) has a great diversity. The result in this paper assumes that the linear system has a compact semigroup and consequently is not completely controllable. Moreover, the functions with Lipschitz condition are considerably strong when one discusses various applications in the real-world problems. Such an assumption is removed from this paper. 


\section{References}

[1] R. P. Agarwal, Y. Zhou, and Y. He, "Existence of fractional neutral functional differential equations," Computers \& Mathematics with Applications, vol. 59, no. 3, pp. 1095-1100, 2010.

[2] J. Tenreiro Machado, V. Kiryakova, and F. Mainardi, "Recent history of fractional calculus," Communications in Nonlinear Science and Numerical Simulation, vol. 16, no. 3, pp. 1140-1153, 2011.

[3] A. A. Kilbas, H. M. Srivastava, and J. J. Trujillo, Theory and Applications of Fractional Differential Equations, Elsevier Science B.V., Amsterdam, The Netherlands, 2006.

[4] J. R. Wang, Y. Zhou, W. Wei, and H. Xu, "Nonlocal problems for fractional integrodifferential equations via fractional operators and optimal controls," Computers \& Mathematics with Applications, vol. 62, no. 3, pp. 1427-1441, 2011.

[5] Z. Liu and X. Li, "On the controllability of impulsive fractional evolution inclusions in Banach spaces," Journal of Optimization Theory and Applications, vol. 156, no. 1, pp. 167-182, 2013.

[6] L. Shen and J. Sun, "Relative controllability of stochastic nonlinear systems with delay in control," Nonlinear Analysis: Real World Applications, vol. 13, no. 6, pp. 2880-2887, 2012.

[7] J. Klamka, "Stochastic controllability of systems with multiple delays in control," International Journal of Applied Mathematics and Computer Science, vol. 19, no. 1, pp. 39-47, 2009.

[8] J. Klamka, "Constrained exact controllability of semilinear systems," Systems \& Control Letters, vol. 47, no. 2, pp. 139-147, 2002.

[9] X. J. Wan, Y. P. Zhang, and J. T. Sun, "Controllability of impulsive neutral functional differential inclusions in Banach spaces," Abstract and Applied Analysis, vol. 2013, Article ID 861568, 8 pages, 2013.

[10] J. Klamka, "Constrained controllability of semilinear systems with delayed controls," Bulletin of the Polish Academy of Sciences, vol. 56, no. 4, pp. 333-337, 2008.

[11] J. Klamka, "Constrained controllability of semilinear systems with delays," Nonlinear Dynamics, vol. 56, no. 1-2, pp. 169-177, 2009.

[12] Z. Tai and X. Wang, "Controllability of fractional-order impulsive neutral functional infinite delay integrodifferential systems in Banach spaces," Applied Mathematics Letters, vol. 22, no. 11, pp. 1760-1765, 2009.

[13] S. Kumar and N. Sukavanam, "Approximate controllability of fractional order semilinear systems with bounded delay," Journal of Differential Equations, vol. 252, no. 11, pp. 6163-6174, 2012.

[14] A. Debbouche and D. Baleanu, "Controllability of fractional evolution nonlocal impulsive quasilinear delay integro-differential systems," Computers \& Mathematics with Applications, vol. 62, no. 3, pp. 1442-1450, 2011.

[15] J. Klamka, "Local controllability of fractional discrete-time semilinear systems," Acta Mechanica et Automatica, vol. 5, no. 2, pp. 55-58, 2011.

[16] J. Klamka, "Controllability and minimum energy control problem of fractional discrete-time systems," in New Trends in Nanotechnology and Fractional Calculus Applications, D. Baleanu, Z. B. Guvenc, and J. A. Tenreiro Machado, Eds., pp. 503-509, Springer, New York, NY, USA, 2010.

[17] R. Sakthivel, N. I. Mahmudov, and J. J. Nieto, "Controllability for a class of fractional-order neutral evolution control systems," Applied Mathematics and Computation, vol. 218, no. 20, pp. 10334-10340, 2012.
[18] N. I. Mahmudov and A. Denker, "On controllability of linear stochastic systems," International Journal of Control, vol. 73, no. 2, pp. 144-151, 2000.

[19] P. Muthukumar and P. Balasubramaniam, "Approximate controllability of mixed stochastic Volterra-Fredholm type integrodifferential systems in Hilbert space," Journal of the Franklin Institute, vol. 348, no. 10, pp. 2911-2922, 2011.

[20] R. Sakthivel, Y. Ren, and N. I. Mahmudov, "On the approximate controllability of semilinear fractional differential systems," Computers \& Mathematics with Applications, vol. 62, no. 3, pp. 1451-1459, 2011.

[21] R. Sakthivel, J. J. Nieto, and N. I. Mahmudov, "Approximate controllability of nonlinear deterministic and stochastic systems with unbounded delay," Taiwanese Journal of Mathematics, vol. 14, no. 5, pp. 1777-1797, 2010. 


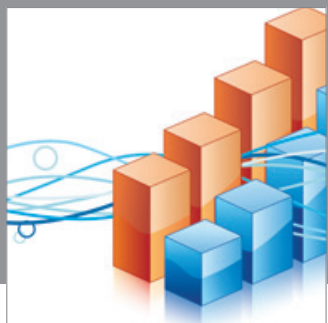

Advances in

Operations Research

mansans

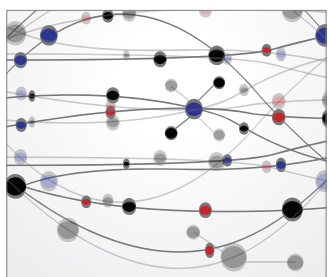

The Scientific World Journal
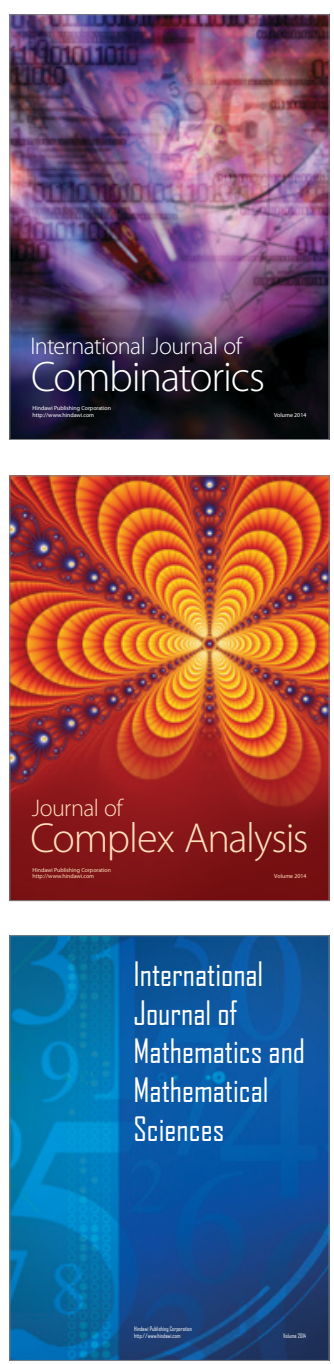
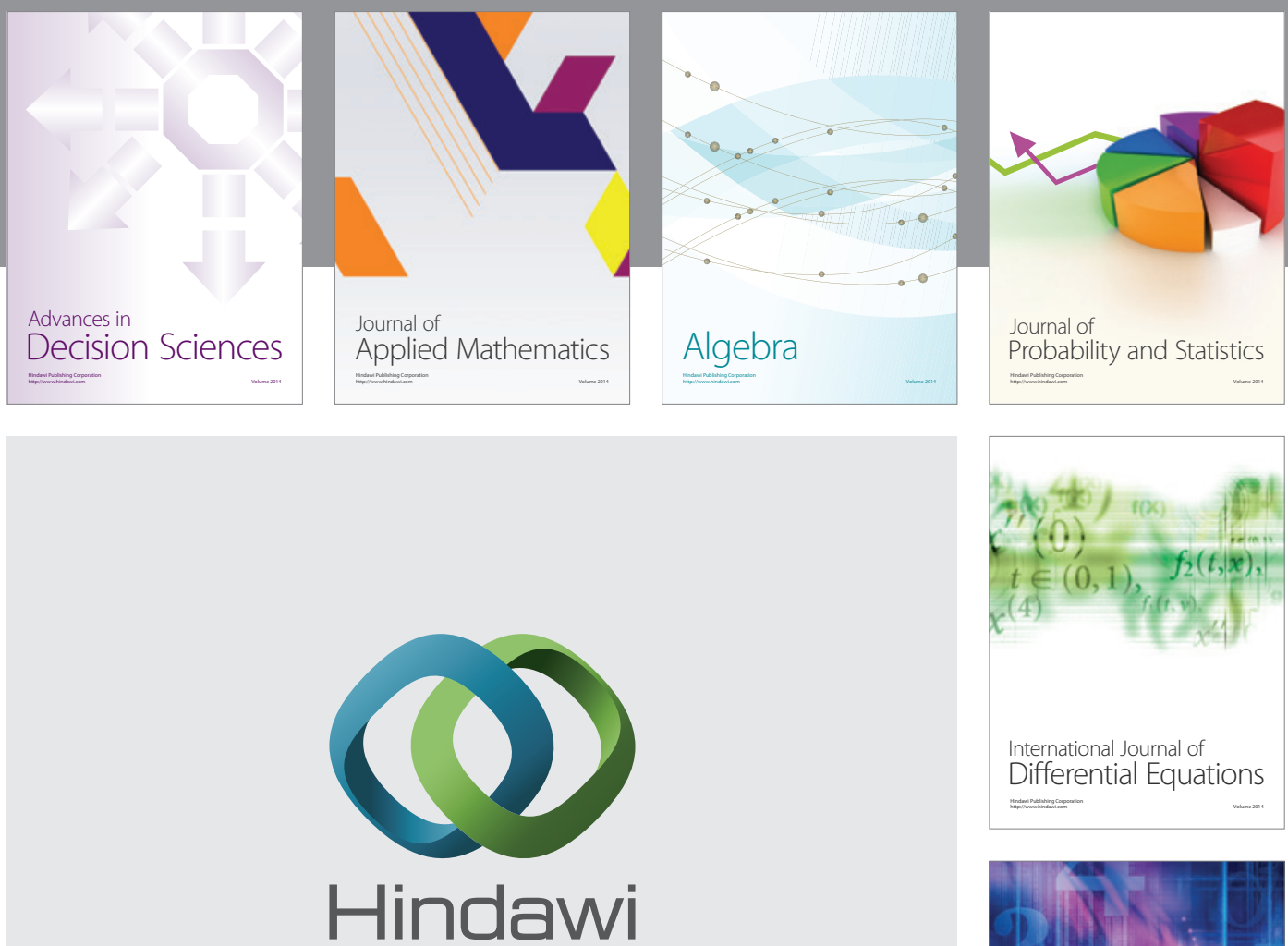

Submit your manuscripts at http://www.hindawi.com
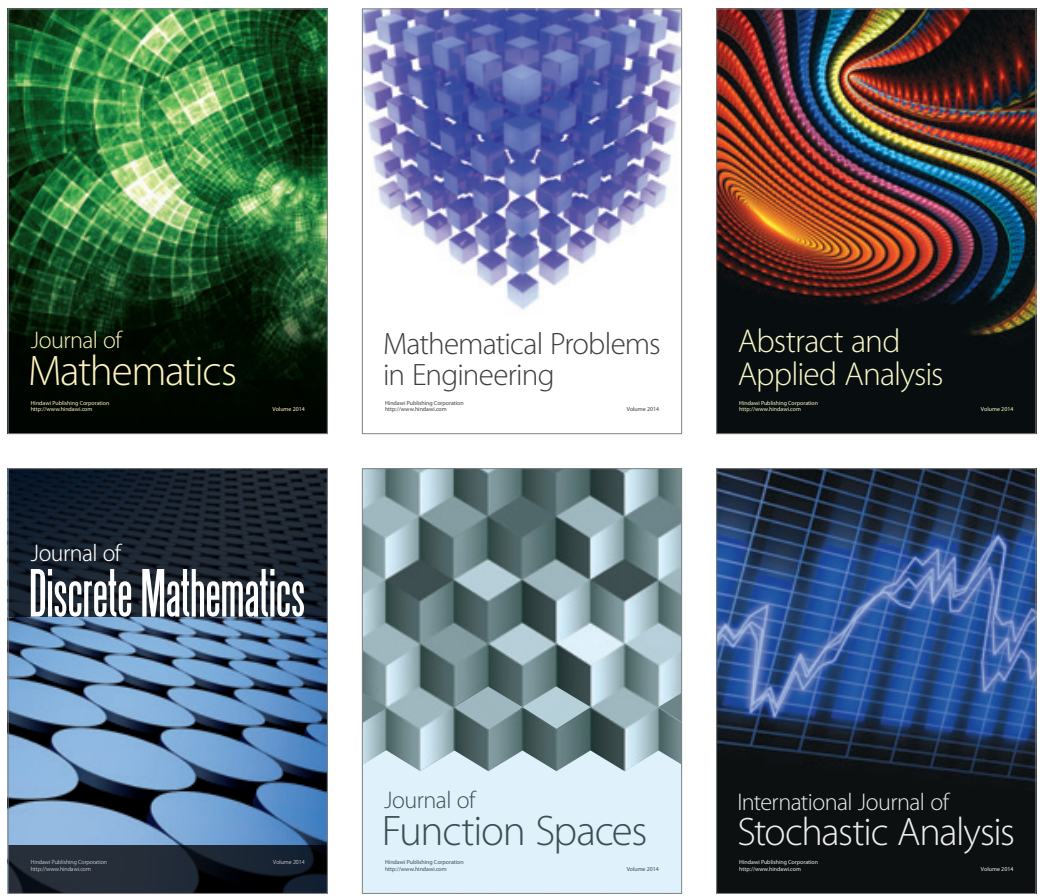

Journal of

Function Spaces

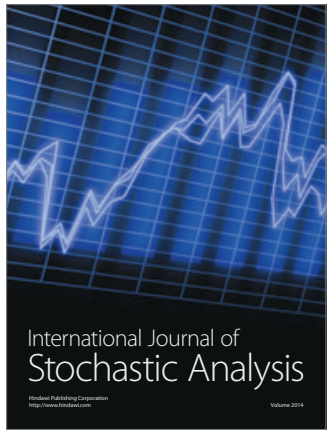

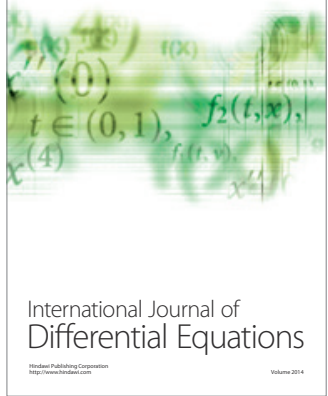
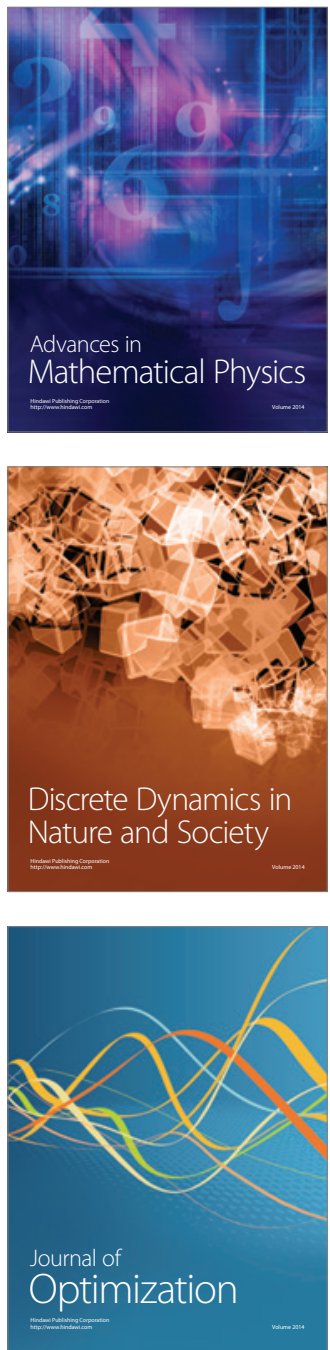